\title{
Genetic variability in the case of COVID-19 infection
}

Undoubtedly, coronavirus disease 2019 (COVID-19), which is caused by severe acute respiratory syndrome coronavirus 2 (SARS$\mathrm{CoV}-2)$, has been the most challenging outbreak so far in relation to severe acute respiratory syndrome coronavirus (SARS-CoV) and Middle East respiratory syndrome coronavirus (MERS$\mathrm{CoV})$. One of the lessons of the current pandemic is the high transmission rate and large variations in disease severity; so, it may be suggested that genetic variability may be involved in immune response and symptom severity. ${ }^{1}$ For this reason, in this article, we describe the genetic aspects presently under study that may have an impact on the different clinical presentations of SARSCoV-2.

Initially, one of the aspects to be described are genetic polymorphisms existing in the ACE2 gene (OMIM 300335), which encodes the cellular receptor for COVID-19. Allelic variants of this gene may influence the protein's binding with the virus and subsequent invasion of the cell. In addition, polymorphisms of cellular proteases may facilitate the entry of virus into the cell, along with furin and TMPRSS2, a transmembrane serine protease $2 .{ }^{1}$ Variants in the latter have also been considered in infection severity. ${ }^{2}$ For example, a study conducted in approximately 81000 human genomes found that ACE2 polymorphism p.Arg514Gly, present in the African/African-American population, was associated with cardiovascular and pulmonary conditions and that polymorphism p.Val160Met (rs12329760) in TMPRSS2 was a potential candidate for differential genetic susceptibility to COVID-19 as well as for risk factors, including cancer and the high-risk group of male patients. ${ }^{3}$ In addition, variants in gene $A C E 2$, including K31R, N33I, H34R, E35K, E37K, D38V, Y50F, N51S, M62V, K68E, F72V, Y83H, G326E, G352V, D355N, Q388L, and D509Y, have a binding affinity for SARS-CoV-2 and may play a role in resistance to COVID-19. However, these variants are highly uncommon. ${ }^{4}$

On the other side, an association has been suggested with the genetic variability across the three major histocompatibility complex (MHC) class I genes (human leukocyte antigen A [HLA-A], $-B$, and - $C$ genes). Specifically, the $H L A-B * 46: 01$ gene product has the fewest binding peptides for COVID-19, suggesting that individuals with this allele may be more vulnerable to COVID-19 due to their reduced ability to present the viral antigen on immune cells. Conversely, it has been found that the protein encoded by $H L A-B{ }^{*} 15: 03$ showed the greatest capacity to present highly conserved SARS-CoV-2 peptides that are shared among common human coronaviruses, ${ }^{5}$ suggesting that it could enable cross-protective T-cell-based immunity, ${ }^{5}$ so people carrying this genotype may be more prone to developing immunity. ${ }^{5}$ These results have been demonstrated in previous studies on SARS-CoV. ${ }^{5}$ Another study has shown that HLA-Cw*1502 and $D R B 1^{*} 0301$ may act as resistance factors in SARS-CoV infection. In addition, $H L A-B^{*} 0703$ has displayed a significant rise in the SARS-CoV patient group. ${ }^{6}$ Therefore, the association between the HLA genotype and disease severity may be extended to other unrelated viruses. After the development of a vaccine, individuals with high-risk HLA types should be prioritized for immunization. ${ }^{5}$

In addition, the genes regulating Toll-like receptor and complement pathways and subsequently cytokine storm induced exaggerated inflammatory pathways seem to underlie the severity of COVID-19; and these genes may account for a third option, after ACE2 and HLA mentioned above. ${ }^{7}$

Also, there is the ADF/ cofilin complex, composed of ADF, actin-depolymerizing factor, which is encoded by the DSTN gene (OMIM 609114), and cofilin, an actin-modulating protein encoded by CFL1 (OMIM 601442) and CFL2 (OMIM 601443). In the initial stages of viral infection, hyperactivation of cofilin and inefficient actin polymerization are known to occur. For this reason, the allelic variants in these three genes, as well as the ACE2 gene described above, should be further studied in the face of the broad range of phenotypes described for SARS-CoV- $2 .{ }^{1}$

Genome-wide association studies (GWAS) were performed in an attempt to delineate host genetic factors contributing to the severe clinical presentation of COVID-19 characterized by respiratory failure in Spanish and Italian patients; 8582968 single-nucleotide polymorphisms were analyzed, and a meta-analysis of the two casecontrol panels was conducted. Cross-replicating associations with rs11385942 at locus 3p21.31 and with rs657152 at locus 9q34.2 were detected, which were significant at the genome-wide level. In the first case, the association signal 
spanned the genes SLC6A20 (OMIM 605616), LZTFL1 (OMIM 606568), CCR9 (OMIM 604738), FYCO1 (OMIM 607182), CXCR6 (OMIM 605163), and XCR1 (OMIM 60055). On its side, the association signal at locus $9 \mathrm{q} 34.2$ coincided with the ABO blood group locus (OMIM 616093). A blood-group-specific analysis showed a higher risk in blood group A than in other blood groups and a protective effect in blood group $\mathrm{O}$ as compared with other blood groups. ${ }^{8}$ However, another study indicated that patients with $B$ and $\mathrm{AB}$ blood group had more possibilities of testing positive for COVID-19, whereas those with O group had lower chances. On their side, patients with a positive $\mathrm{Rh}$ factor were more likely to test positive. ${ }^{9}$

The understanding of genetic susceptibility to infection and its severity are only just beginning. Genome sequencing in selected patients may find single-nucleotide variant differences in possibly relevant genes or genomic regions in the afflicted and the asymptomatic groups. ${ }^{1}$ The scientific community is trying to address this issue by combining research efforts using existing genetic databases to turn them into active areas of investigation. Elucidation of genomics and genetic pathways related to COVID-19 susceptibility might aid clinicians in dealing with possible future waves of this pandemic. ${ }^{1}$

There remain substantial unanswered questions regarding the role of individual genetic variability in the immune response against SARSCoV-2. We hypothesize that individual HLA genotypes may differentially induce the T-cell mediated antiviral response and could potentially alter the course of disease and its transmission. ${ }^{5}$

It is expected that the genetic aspects involved in COVID-19 infection susceptibility and prognosis are multifactorial, and the combination of all these findings contributed by the international scientific community will be helpful to better understand these aspects, which may then offer special support to those individuals with higher susceptibility and risk levels.

Prof. Francisco Cammarata-Scalisi, M.D., MSc. ${ }^{a}$ Prof. Antonio Cárdenas Tadich, M.D., PhD. ${ }^{a}$ Michele Callea, DDS, MoH, MSc. ${ }^{b}$

a. Department of Pediatrics, Hospital Regional de Antofagasta, Chile.

b. Unit of Dentistry, Bambino Gesù Children's Hospital, IRCCS, Rome, Italy.

http: / / dx.doi.org/10.5546/ aap.2020.eng.304

To cite: Cammarata-Scalisi F, Cárdenas Tadich A, Callea M. Genetic variability in the case of COVID-19 infection. Arch Argent Pediatr 2020;118(5):304-305.

\section{REFERENCES}

1. Godri PollittKJ, Peccia J, Ko AI, Kaminski N, et al. COVID-19 vulnerability: the potential impact of genetic susceptibility and airborne transmission. Hum Genomics. 2020;14(1):17.

2. Asselta R, Paraboschi EM, Mantovani A, Duga S. ACE2 and TMPRSS2 variants and expression as candidates to sex and country differences in COVID-19 severity in Italy. Aging (Albany NY). 2020:15(11):10087-98.

3. Hou Y,ZhaoJ, Martin W, Kallianpur A, et al. New insightsinto genetic susceptibility of COVID-19: an ACE2 and TMPRSS2 polymorphism analysis. BMC Med. 2020;18(1):216.

4. Stawiski EW, Diwanji D, Suryamohan K, Gupta R, et al. Human ACE2 receptor polymorphisms predict SARSCoV-2 susceptibility. BioRxiv.2020;10.1101/2020.04.07.024752

5. Nguyen A, David JK, Maden SK, Wood M, et al. Human leukocyte antigen susceptibility map for severe acute respiratory syndrome coronavirus 2. J Virol. 2020; 94(13):e00510-20.

6. Mohammadpour S, Torshizi Esfahani A, Halaji M, Lak M, Ranjbar R. An updated review of the association of host genetic factors with susceptibility and resistance to COVID-19. J Cell Physiol. 2020;10.1002/jcp.29868.

7. Debnath M, Banerjee M, Berk M. Genetic gateways to COVID-19 infection: Implications for risk, severity, and outcomes. FASEB J. 2020;10.1096/fj.202001115R.

8. Ellinghaus D, Degenhardt F, Bujanda L, Buti M, et al. Genomewide Association Study of Severe COVID-19 with Respiratory Failure. N Engl J Med. 2020;NEJMoa2020283.

9. Zhao J, Yang Y, Huang H, Li D, et al. Relationship between the ABO blood group and the COVID-19 susceptibility. MedRxiv. 2020.03.11.20031096. 